\title{
Clinical Profile of COVID-19 Patients Presenting with Uveitis - A Short Case Series
}

\author{
Salam Iriqat $\mathbb{D D}^{\prime}$ \\ Qusai Yousef' \\ Suheir Ereqat ${ }^{2}$ \\ 'Ocular Inflammatory Disease \\ Department, St John Eye Hospital, East \\ Jerusalem, Palestine; ${ }^{2}$ Biochemistry and \\ Molecular Biology Department, Faculty of \\ Medicine, Al-Quds University, East \\ Jerusalem, Palestine
}

\begin{abstract}
Purpose: To present the clinical features of anterior, intermediate and posterior uveitis in patients with COVID-19 and to increase the awareness of the treating physicians to refer patients with COVID-19 who have eye symptoms for ophthalmic exam, in order to diagnose as early as possible and prevent vision-threatening complications.

Methods: Retrospective observational case reports.

Results: We report three cases of COVID-19 patients who developed uveitis during or after the course of their sickness with COVID-19. All patients underwent a detailed eye examination, relevant history and investigations did not prove any other cause of uveitis.

Conclusion: This report presents novel data on the course of subjects with uveitis during the COVID-19 pandemic. Intermediate and posterior uveitis warrant further evaluation with differential diagnosis supported by laboratory tests due to the association with systemic diseases and risk of permanent vision loss. Iridocyclitis, intermediate, and posterior uveitis treatment should be guided by ophthalmologists, particularly uveitis specialists, when possible.
\end{abstract}

Keywords: SARS-CoV-2, uveitis, COVID-19

\section{Introduction}

Severe acute respiratory syndrome coronavirus 2 (SARS-COV-2) is an enveloped, positive sense, RNA virus, belonging to the family Coronaviridae, genus Betacoronavirus, was first identified and reported in Wuhan, the capital city of Hubei province in China, in December, 2019 and reported as the causative agents of the severe respiratory illness termed COVID-19. ${ }^{1}$ At the time of writing this paper there have been over 122 million confirmed COVID-19 cases, 98 million recovered, with over 2.7 million deaths globally. ${ }^{2}$ In Palestine, data obtained from the Palestinian ministry of health registration showed 230,609 confirmed COVID-19 cases, 205,920 recovered cases, and over 2449 deaths. $^{3}$

Uveitis is an autoimmune disease of the eye that refers to any of a number of intraocular inflammatory conditions. It is reported that patients with uveitis who are taking high doses of corticosteroids and immunomodulatory therapies (IMT) are considered at high risk to get infected with severe COVID-19 disease. ${ }^{4,5}$ A caseseries of 18 hospitalized patients identified SARS-CoV-2 RNA in tear samples in 28\% of confirmed COVID-19 patients indicating that the respiratory tract is not the only transmission route. ${ }^{6}$ However, the absence of ocular signs in patients with positive tear samples imply that viral shedding in tears is not always related to ocular inflammation. ${ }^{7}$ Viral infection of the conjunctiva could induce a local immune or
Correspondence: Salam Iriqat Email Salam.erakat@sjeh.org 
inflammatory response resulting in ocular manifestations. Cell-mediated immunity can play a major role in viral diseases in which infected cells in the uveal and corneal tissue have viral-specific antigens on their surface. Cell-mediated immunity can cause specific tissue damage, as in acute retinal necrosis. Various immune-regulatory cytokines (IL6 , IL-10, and IFN- $\gamma$ ) were detected in ocular fluid samples from patients with viral uveitis. ${ }^{8}$ Although the CD4+ T lymphocytes drive the disease during the course of experimental uveitis, the number of $\mathrm{CD} 8+\mathrm{T}$ cells is increased indicating that uveitis is the result of much more than the aberrant activation of a single cell type.

Since March 2020, several papers have been published and stressed the risk of COVID-19 transmission among ophthalmologists due to the close contact between the physician and the patient during exam. ${ }^{9}$ Few papers reported conjunctivitis and uveitis in COVID-19 patients, but no cases were reported with findings suggesting that the patient could have been infected with COVID-19 and his eye condition lead to the diagnosis of COVID-19. Most reports mentioned eye complaints associated with COVID-19 including ocular surface disease (pain, redness, and photophobia), few stressed the risk of vision loss due to COVID-19.

We report three cases of COVID-19 patients with severe uveitis. These cases have not been reported previously and have been approved by the ethics committee of St John Eye Hospital (East Jerusalem, Palestine). All patients gave informed consent for the publication of their case details and any accompanying images. Herein, we stress on the importance of urgent ophthalmic assessment of any COVID-19 patient complaining of any eye symptoms (redness, pain, tearing, conjunctival swelling, blurred vision, pain with ocular movement), and highlight the professional role of ophthalmologists in managing COVID-19 patients with signs of uveitis.

\section{Case I}

A 19-year-old male patient, with no significant past medical history, wearing glasses for myopia, presented to $\mathrm{St}$ John Eye Hospital complaining of both eyes redness and a blurred vision of one-month duration following recovery from COVID-19 (Table 1). The patient tested positive for SARS-CoV-2 by RT-PCR obtained from a nasopharyngeal swab on Dec 1, 2020 (Table 2). He was suffering from fever with a temperature of $\left(41^{\circ} \mathrm{C}\right)$, cough, headache, joint pain, and skin rash (Table 1). One month later he developed pneumonia, he was treated as an outpatient by systemic oral antibiotics (azithromycine $500 \mathrm{mg}$ daily for one week and oral dexamethasone (6 $\mathrm{mg}$ daily for five days). The patient started to complain of blurred vision, redness, and pain in both eyes five weeks post-positive PCR testing. At the initial examination on Jan 28, 2021, his bestcorrected visual acuity was $6 / 18$ in the right eye and $6 / 60$ in his left eye. Slit-lamp examination of the right eye showed conjunctival hyperemia, corneal edema and endothelial KPs (keratic precipitates), anterior chamber inflammatory cells with normal fundus examination. Left eye examination showed signs of more aggressive

Table I Systemic Symptoms, Ocular Findings, Classification of Uveitis and Given Medication

\begin{tabular}{|l|c|c|c|}
\hline Demographic Data & Case I & Case 2 & Case 3 \\
\hline Age (year) & 19 & 29 & 62 \\
\hline Gender & Male & Male & Male \\
\hline Eye and systemic symptoms & Both eyes & Both eyes & No eye \\
Redness & Both eyes & Both eyes \\
Photophobia & Both eyes & Both eyes & Right eye \\
Blurred vision & Both eyes & Noth eyes \\
Lacrimation & Yes & Yes & No \\
Loss of taste and or smell & $41^{\circ} \mathrm{C}$ & Yes & No \\
Fatigue & Yilateral anterior uveitis & Bilateral intermediate and & $36.5^{\circ} \mathrm{C}$ \\
Fever & & posterior uveitis & Right eye iridocyclitis \\
\hline Classification of uveitis & Topical and systemic steroid & Topical and systemic steroid & Cyclopentolate \\
\hline Cyclopentolate & Cyclopentolate \\
\hline
\end{tabular}


Table 2 Relevant Laboratory Tests

\begin{tabular}{|c|c|c|c|c|}
\hline Laboratory Tests & Case I & Case 2 & Case 3 & Normal Values \\
\hline SARS-CoV-2 RT-PCR & Positive & ND & Positive & \\
\hline Leucocytes & 13.2 & 7.8 & 6 & $4000-10,000 / \mu \mathrm{L}$ \\
\hline ESR & 47 & 5 & 12 & $0-20 \mathrm{~mm} / \mathrm{h}$ \\
\hline CRP & 55 & 7 & 4 & Less than $10 \mathrm{mg} / \mathrm{L}$ \\
\hline RF & 5 & 6 & 3 & Less than $14 \mathrm{IU} / \mathrm{mL}$ \\
\hline ALT & 14 & 20 & 22 & $7-55$ U/L \\
\hline AST & 18 & 22 & 28 & $8-48 \mathrm{U} / \mathrm{L}$ \\
\hline Creatinine & I.I & I & I.I & 0.9 to $1.3 \mathrm{mg} / \mathrm{dL}$ \\
\hline ANA & Negative & Negative & Negative & $7-55$ U/L \\
\hline ACE & 10 & 18 & 12 & Less than $40 \mathrm{nmol} / \mathrm{mL} / \mathrm{min}$ \\
\hline HLA B27 & Negative & Negative & Negative & \\
\hline Brucella & Negative & Negative & Negative & \\
\hline PPD & Negative & Negative & Negative & \\
\hline TPHA & ND & Titer $<1: 80$ & ND & Titer $<1: 80$ \\
\hline HIV test & Negative & Negative & Negative & \\
\hline
\end{tabular}

Note: *ND: Not done.

Abbreviations: ESR, erythrocyte sedimentation rate; CRP, C-reactive protein; RF, rheumatoid factor; ALT, alanine aminotransferase; AST, aspartate aminotransferase; ANA, antinuclear antibody; ACE, angiotensin converting enzyme; HLB27, human leukocyte antigen B27; PPD, purified protein derivative; TPHA, treponema pallidum hemagglutination; HIV, human immunodeficiency virus; ND, not done.

behavior with conjunctival hyperemia, corneal edema and $\mathrm{kPs}$, anterior chamber hypopyon, iris deposits over the lens anterior capsule, clear vitreous, and normal fundus examination. Intraocular pressure measured with Goldmann tonometer was $13 \mathrm{~mm} \mathrm{Hg}$ in both eyes. The patient was started on topical steroids (one drop/hour/daily in the first week then slowly tapered by decreasing one drop per day each week till stopping it,) systemic steroids ( $1 \mathrm{mg} / \mathrm{kg}$ ) and cycloplegics, (cyclopentolate 1\%). Laboratory tests to rule out other possible infectious or autoimmune diseases were performed and described in Table 2. The diagnosis of bilateral anterior uveitis secondary to COVID-19 infection was made. On his follow-up visit at the uveitis clinic on Feb 9, 2021, the patient showed a significant improvement, the best-corrected visual acuity was $6 / 12$ in the right eye and $6 / 15$ in the left eye, slit-lamp examination revealed clinical improvement with white conjunctiva, clear cornea, and a quiet and deep anterior chamber. His last follow-up was on May 11, 2021, he is off medications with quiet eye examination and best corrected visual acuity (BCVA) 6/6 in both eyes.

\section{Case 2}

A 29-year-old male patient, wearing glasses for myopia and astigmatism, presented to St John Eye Hospital on Jan 24, 2021 complaining of blurred vision in both eyes and pain of six weeks duration (Table 1), there was no history of joint pain, oral or genital ulcers, or skin rashes. Two months prior to his presentation, the patient suffered from fever, cough, and headache but he ignored his symptoms and did not seek any medical advice, neither PCR nor serology test for COVID-19 were done at that time. At the initial examination, his best-corrected visual acuity was $6 / 18$ in both eyes. Slit-lamp examination for both eyes showed vascular sheathing (Figure 1A), quiet anterior segment, minimal lens opacities, web-like vitreous veils with big white snowballs adherent to the vitreous webs (Figure 1B). Intraocular pressure measured with Goldmann tonometer was $13 \mathrm{mmHg}$ in both eyes. The laboratory tests done for the patient are included in Table 2. Behçet's disease was ruled out (no positive criteria in the history for all the scoring systems). The patient was subjected to COVID-19 antibody test (IgG), six weeks 


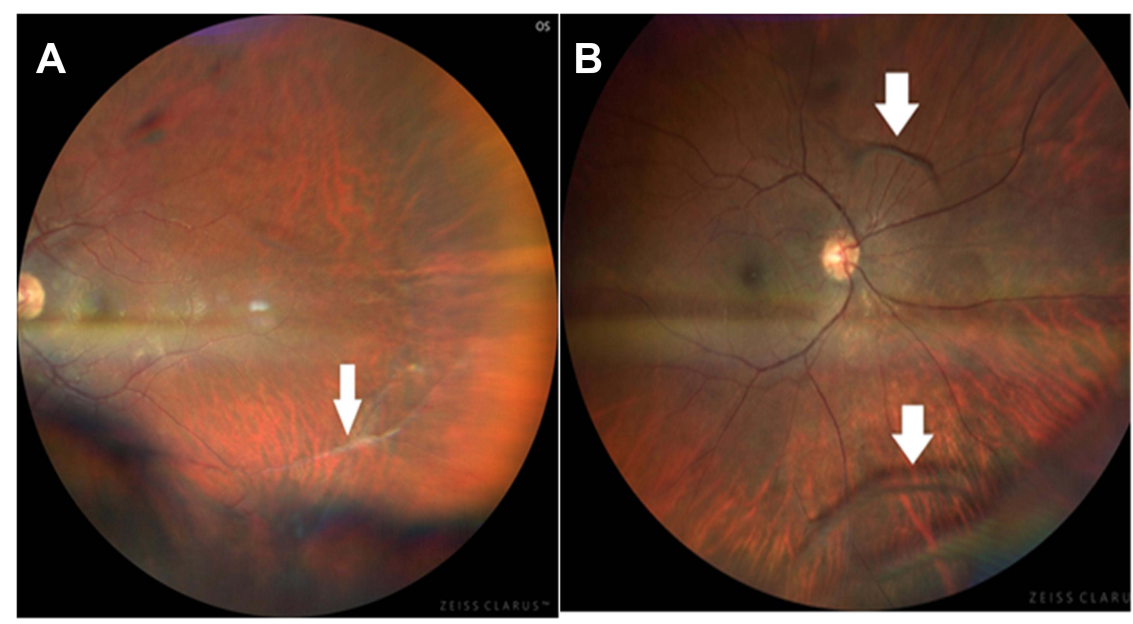

Figure I Color fundus in right (A) and left (B) eyes of 29-year-old male COVID-19 patient demonstrating (A) vascular sheathing indicating vasculitis (B) RE web like vitreous veils indicating intermediate uveitis.

post-development of his viral symptoms, the test came back positive. The diagnosis of intermediate and posterior uveitis secondary to COVID-19 was made after excluding all other possible infectious and noninfectious causes.

The patient was treated with prednisone $1 \mathrm{mg} / \mathrm{kg}$ for one week then tapered by $5 \mathrm{mg}$ each week through his follow-up period, topical steroid pred forte (prednisolone acetate) was given every hour during the first week then tapered slowly. During his follow-up visits in February and March 2021, patient was doing well, his bestcorrected visual acuity improved to 6/7.5 in both eyes. On April 8, 2021, the patient presented to the clinic with flare up in the right eye and increased vitreous opacity while he was on prednisone $5 \mathrm{mg}$ daily. Leptospira immunoglobulin test was requested in April 2021-after the paper submission - but unfortunately is not available in Palestine and due to COVID-19 pandemic restrictions, we could not send him to do the test in nearby countries, However, he was discharged on prednisone $1 \mathrm{mg} / \mathrm{kg}$, Imuran $^{\mathrm{TM}}$ (azathioprine), steroid sparing agent was added. On May 6, 2021, the patient was on prednisone $20 \mathrm{mg}$ daily, azathioprine $50 \mathrm{mg}$ twice daily, with significant improvement upon examination.

\section{Case 3}

A 62-year-old male patient, with no significant past medical history and known ocular history of right eye primary angle-closure glaucoma controlled by COSOPT ${ }^{\circledR}$ eye drops (timolol $0.5 \%$, dorzolamide $2 \%$ ), He underwent right eye phacoemulsification and intraocular lens implantation on Nov 29, 2020, due to a right eye cataract. On
Dec 28, 2020, he tested positive for COVID-19 by RTPCR. He did not complain of any respiratory symptoms and all blood tests were normal (Table 2). The only complaint was recurrent attacks of right eye pain, redness, and blurred vision with no photophobia. He was referred to our uveitis clinic on Feb 5, 2021, for further investigations. At the initial examination, his best-corrected visual acuity was $6 / 9$ in the right eye and 6/6 in his left eye. Slit-lamp examination was remarkable for right eye mild conjunctival hyperemia, with multiple corneal keratic precipitates (KPs) (Figure 2, white arrow), and a deep anterior chamber with +4 cells (Figure 2, yellow arrows). Fundus examination revealed a clear vitreous and flat healthy retina. Intraocular pressure measured with Goldmann tonometer was $11 \mathrm{mmHg}$ in both eyes. Under the diagnosis of right eye iridocyclitis mostly secondary to COVID-19 infection,

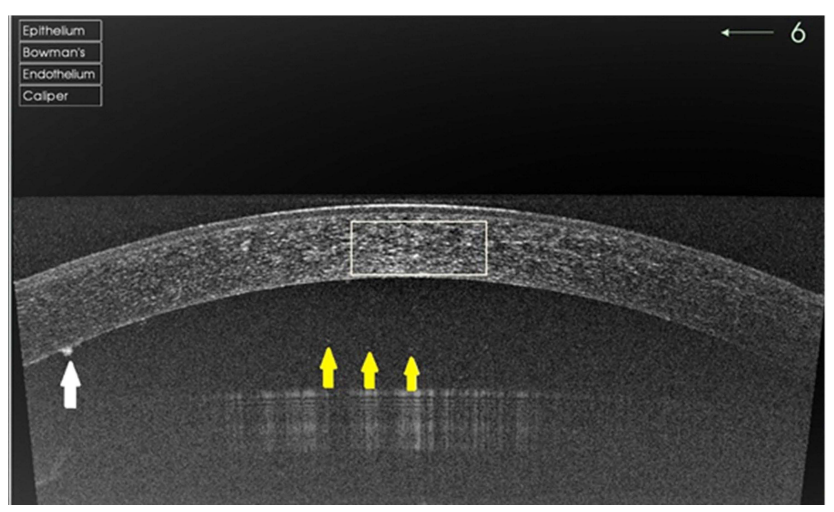

Figure 2 Slit-lamp examination eyes of 62-year-old male COVID-19 patient demonstrating endothelial keratic precipitate (white arrow), cells in the anterior chamber indicating iridocyclitis (yellow arrows). 
the patient was treated with topical steroids (dexamethasone $1 \mathrm{mg} / \mathrm{mL}$ ) one drop/hour/daily in the first week then slowly tapered by decreasing one drop per day each week till stopping it, cyclopentolate $1 \%$ was instilled three times daily for 1 week then stopped. Seven days later, the patient showed a significant improvement, the best-corrected visual acuity was $6 / 7.5$ in the right eye, slit-lamp examination revealed clinical improvement with white conjunctiva, clear cornea with few KPs, and a few cells in the anterior chamber. The plan was to stop cycloplegics, tapering steroids, and to continue his follow-up in the glaucoma clinic.

\section{Discussion}

To the best of our knowledge, this is the first clinical report of bilateral intermediate and posterior uveitis due to COVID-19 (as seen in case 2). It is well known that SARS-CoV-2 induces conjunctivitis. A study conducted on 38 COVID-19 infected patients showed that $5 \%$ of them had virus-positive conjunctivitis and $32 \%$ had conjunctivitis. ${ }^{10}$ Moreover, retinal hemorrhages, central retinal vein occlusion and other retinal thrombotic complications have been observed in COVID-19 patients. ${ }^{11,12}$

Here, we described three cases of uveitis developing a few weeks after diagnosis of COVID-19, new mutations of the virus are still emerging which should raise red flags for possibly more severe complications and higher rates of infection. The COVID-19 pathophysiology is still not well understood as many factors affect the prognosis. Patients controlled with immunosuppressive medications showed milder degrees of inflammation than those who are not receiving any medication. Uveitis patients who are treated with systemic immunosuppression - to control ocular inflammation - are potentially at higher risk of COVID19 infection. ${ }^{5,13}$ Current perspectives are to use corticosteroids at the minimum possible dose for autoimmune conditions in the absence of COVID-19. ${ }^{14}$ However, according to the World Health Organization (WHO) recommendations; systemic corticosteroids should be avoided in severe cases of COVID-19. ${ }^{15}$ A clinical trial conducted in the UK showed that low dose dexamethasone can reduce the mortality of COVID-19 patients. ${ }^{16}$ Yet, the international experts and specialists have controversial opinions regarding the use of corticosteroids in COVID19 patients. As we described in case 1, a diagnosis of bilateral anterior uveitis secondary to COVID-19 infection was made in a 19-year-old male patient who suffered from pneumonia. The ocular findings of this patient improved following topical and systemic corticosteroid therapy which was tapered over a period of six weeks. A recent case report ${ }^{17}$ showed that early use of corticosteroids at a moderate dose for a short course may enhance recovery of a COVID-19 patient. In this context, we support timely and appropriate use of corticosteroids in COVID-19 cases taking into consideration uveitis severity, systemic comorbidities, age, and lens status. The diagnosis of bilateral intermediate and posterior uveitis was made in an apparently healthy man (case 2) with no previous systemic illnesses, upon his ocular findings of vascular sheathing as a sign of posterior uveitis, and peripheral inferior vitreous condensations (snowballs) as a sign of intermediate uveitis. Taking into consideration his viral prodromal symptoms prior to his eye disease made us think of infectious uveitis, while these days the pandemic is spreading enormously in Palestine, we asked the patient to do coronavirus IgG titer which returned positive, and all other tests returned negative leaving COVID-19 as the presumptive etiology for the inflammation. Intermediate and posterior uveitis warrant further evaluation with differential diagnosis supported by laboratory tests due to the association with systemic diseases and risk of permanent vision loss.

This case report described the significance of ocular symptoms which can be overlooked if patients do not show typical systemic symptoms of COVID-19. The cytokine storm is the leading side effect during cellular immunotherapy, which can be triggered by viral infections. By treating this patient, studying his presentation, we strongly stress the importance of detailed medical history of the patient. Alternatively, conjunctiva is considered as an important portal of entry for respiratory viruses, conjunctival secretions may contain virus and spread viral infection. $^{18}$ The close proximity between patients and ophthalmologists during slit lamp examination may pose an infectious risk to ophthalmologists. In their study, Lai et al suggested a variety of control measures that may be adopted to reduce the risk of coronavirus transmission in ophthalmic facilities. ${ }^{13}$ Moreover, the American Academy of Ophthalmology has issued an important alert for ophthalmologists advising them to wear protective eye masks when seeing conjunctivitis patients with respiratory symptoms. $^{19}$

In case 3 , our patient had right eye cataract surgery one month prior to his COVID-19 confirmed illness, with previous ocular history of well-controlled glaucoma in his right eye. Our patient had well-controlled primary angle 
closure glaucoma for two years with topical antiglaucoma treatment but a few weeks after the surgery, he developed iridocyclitis as a consequence of COVID-19 infection. It is well known that people with preexisting health conditions are more vulnerable to infection and the efficacy of virus entry into host cells partially depends on the immune conditions of the host and the presence of $A C E 2$ on host cell membrane. ${ }^{20}$ Previous reports showed that the human conjunctival, corneal and limbal epithelial cell, retina, respiratory tract, enterocytes of the small intestine, and the proximal tubules of the kidney share the same $A C E 2$ receptors for COVID-19 virus. ${ }^{21}$ It is reported that a transmembrane serine protease (TMPRSS2) cleaves the $\mathrm{S}$ protein into $\mathrm{S} 1$ and $\mathrm{S} 2$ domains upon virus binding to $A C E 2$ and thus enables fusion of the viral and host cellular membranes allowing the viral entry. Cornea is also considered a potential site for SARS-CoV-2 infection since coexpression of $A C E 2$ and TMPRSS2 were reported in corneal epithelium and endothelium. However, $A C E 2$ expression in ocular surface is much lower than in lung and kidney tissues as only $6.6 \%$ of cells of the superficial conjunctival epithelium express both entry proteins TMPRSS2 and ACE2. ${ }^{22,23}$ Hence, the ability of SARS$\mathrm{CoV}$ spike protein to bind with conjunctival $A C E 2$ is much lower than that in lung cells. ${ }^{24}$ Moreover, the tear film and the fast drainage may provide a barrier for infection of the underlying epithelia preventing the viral persistence and binding to the cornea and/or conjunctival epithelial receptors. ${ }^{25}$ Nevertheless, the potential for systemic transmission from the nasolacrimal duct cannot be ruled out.

During the pandemic, patients undergoing surgery should be closely followed-up and monitored to avoid any complication. The use of eye prophylaxis in our clinical practice protocol is highly recommended to prevent the spread of the virus through the ocular route of transmission. COVID-19 should be considered in the differential diagnosis while dealing with any patient suffering from eye complaints following surgery or being treated for previous ocular disease.

In conclusion, we presented ocular involvement in three COVID-19 cases to emphasize the importance for the health-care providers to be aware of the possible inflammatory ocular manifestation during and after COVID-19 infection. All physicians should keep in mind the possibility of uveitis in the differential diagnosis when dealing with patients who had previous viral prodromal symptoms up to a few weeks prior to presenting with uveitis.

\section{Acknowledgments}

The authors thank the patients who agreed to participate in this report.

\section{Disclosure}

The authors report no conflicts of interest and no financial support for this work.

\section{References}

1. Wang C, Horby PW, Hayden FG, Gao GF. A novel coronavirus outbreak of global health concern. Lancet. 2020;395 (10223):470-473. doi:10.1016/S0140-6736(20)30185-9

2. Worldmeter. COVID-19 Coronavirus Pandemic. Available from: https://www.worldmeters.info/coronavirus/. Accesssed June 10, 2021.

3. Palestinian Ministry of Health records smp. 2020.

4. Agarwal AK, Sudharshan S, Mahendradas P, et al. Impact of COVID-19 pandemic on uveitis patients receiving immunomodulatory and biological therapies (COPE STUDY). Br J Ophthalmol. 2020.

5. Hung JCH, Li KKW. Implications of COVID-19 for uveitis patients: perspectives from Hong Kong. Eye (Lond). 2020;34(7):1163-1164. doi:10.1038/s41433-020-0905-1

6. Dutescu RM, Banasik P, Schildgen O, Schrage N, Uthoff D. Detection of Coronavirus in tear samples of hospitalized patients with confirmed SARS-CoV-2 from oropharyngeal swabs. Cornea. 2020.

7. Arora R, Goel R, Kumar S, et al. Evaluation of SARS-CoV-2 in tears of patients with moderate to severe COVID-19. Ophthalmology. 2021;128(4):494-503. doi:10.1016/j.ophtha.2020.08.029

8. Balamurugan S, Das D, Hasanreisoglu M, et al. Interleukins and cytokine biomarkers in uveitis. Indian J Ophthalmol. 2020;68 (9):1750. doi:10.4103/ijo.IJO_564_20

9. Mazzotta C, Giancipoli E. Anterior acute uveitis report in a SARS-CoV-2 patient managed with adjunctive topical antiseptic prophylaxis preventing 2019-nCoV spread through the ocular surface route. Int Med Case Rep J. 2020;13:513-520. doi:10.2147/IMCRJ. S260252

10. Wu P, Duan F, Luo C, et al. Characteristics of ocular findings of patients with Coronavirus disease 2019 (COVID-19) in Hubei Province, China. JAMA Ophthalmol. 2020;138(5):575-578. doi:10.1001/jamaophthalmol.2020.1291

11. Walinjkar JA, Makhija SC, Sharma HR, Morekar SR, Natarajan S. Central retinal vein occlusion with COVID-19 infection as the presumptive etiology. Indian $J$ Ophthalmol. 2020;68(11):2572. doi:10.4103/ijo.IJO_2575_20

12. Duff SM, Wilde M, Khurshid G. Branch retinal vein occlusion in a COVID-19 positive patient. Cureus. 2021;13(2). doi:10.7759/ cureus. 13586

13. Lai THT, Tang EWH, Chau SKY, Fung KSC, Li KKW. Stepping up infection control measures in ophthalmology during the novel coronavirus outbreak: an experience from Hong Kong. Graefes Arch Clin Exp Ophthalmol. 2020;258(5):1049-1055. doi:10.1007/s00417-02004641-8

14. Grabbe S, Beissert S, Enk A. Systemic immunosuppression in times of COVID-19: do we need to rethink our standards? $J$ Dtsch Dermatol Ges. 2020;18(8):810-813.

15. World Health Organization. Coronavirus disease (COVID-19): HIV and antireretrovirals. Available from: https://www.who.int/newsroom/q-a-detail/q-a-on-covid-19-hiv-and-antiretrovirals. Accessed June 10, 2021. 
16. Ledford H. Coronavirus breakthrough: dexamethasone is first drug shown to save lives. Nature. 2020;582(7813):469. doi:10.1038/ d41586-020-01824-5

17. Wang K, Tan F, Zhou R, et al. Therapeutic response to corticosteroids in a critically ill patient with COVID-19: a case report. Medicine (Baltimore). 2020;99(31):e21597. doi:10.1097/MD.0000000000021597

18. Belser JA, Rota PA, Tumpey TM. Ocular tropism of respiratory viruses. Microbiol Mol Biol Rev. 2013;77(1):144-156. doi:10.1128/ MMBR.00058-12

19. Ophthalmology AAo. Alert: important coronavirus context for ophthalmologists; 2020.

20. Sun CB, Wang YY, Liu GH, Liu Z. Role of the eye in transmitting human coronavirus: what we know and what we do not know. Front Public Health. 2020;8:155. doi:10.3389/fpubh.2020.00155

21. Hamming I, Timens W, Bulthuis ML, Lely AT, Navis G, van Goor H. Tissue distribution of ACE2 protein, the functional receptor for SARS coronavirus. A first step in understanding SARS pathogenesis. J Pathol. 2004;203(2):631-637. doi:10.1002/path.1570
22. Collin J, Queen R, Zerti D, et al. Co-expression of SARS-CoV-2 entry genes in the superficial adult human conjunctival, limbal and corneal epithelium suggests an additional route of entry via the ocular surface. Ocul Surf. 2021;19:190-200. doi:10.1016/j.jtos.2020.05.013

23. Ma D, Chen CB, Jhanji V, et al. Expression of SARS-CoV-2 receptor ACE2 and TMPRSS2 in human primary conjunctival and pterygium cell lines and in mouse cornea. Eye (Lond). 2020;34(7):1212-1219. doi:10.1038/s41433-020-0939-4

24. Chen X, Yu H, Mei T, et al. SARS-CoV-2 on the ocular surface: is it truly a novel transmission route? Br J Ophthalmol. 2020:bjophthalmol-2020-316263. doi:10.1136/bjophthalmol-2020-316263

25. Armstrong L, Collin J, Mostafa I, Queen R, Figueiredo FC, Lako M. In the eye of the storm: SARS-CoV-2 infection and replication at the ocular surface? Stem Cells Transl Med. 2021. doi:10.1002/sctm.20-0543

\section{Publish your work in this journal}

The International Medical Case Reports Journal is an international, peer-reviewed open-access journal publishing original case reports from all medical specialties. Previously unpublished medical posters are also accepted relating to any area of clinical or preclinical science. Submissions should not normally exceed 2,000 words or 4 published pages including figures, diagrams and references. The manuscript management system is completely online and includes a very quick and fair peer-review system, which is all easy to use. Visit http://www.dovepress.com/testimonials.php to read real quotes from published authors. 